\title{
Hemiballismus in Patients With Poorly Controlled Type 2 Diabetes Mellitus
}

Pressley Chakales, MD; Ariel Park; Austin Amos, MS; Esther Baldinger, MD; Igor Sirotkin, MD; and Alfred Frontera, MD

\begin{abstract}
Swift identification of hemiballismus as a complication of poorly controlled type 2 diabetes mellitus may help facilitate optimal care through glycemic control and prevent debilitating loss of patients' function and autonomy, prolonged hospital stays, and overuse of resources.
\end{abstract}

\author{
Austin Amos and Ariel \\ Park are Medical Students, \\ Pressley Chakales was a \\ Medical Student at the time \\ the article was written; \\ Esther Baldinger and \\ Alfred Frontera are \\ Associate Professors of \\ Neurology; and Igor Sirotkin \\ is an Assistant Professor of \\ Neurology; all at the \\ University of Central Florida \\ College of Medicine in \\ Orlando. Igor Sirotkin is an \\ Assistant Professor of \\ Radiology at the \\ University of Southern \\ Florida in Tampa. Esther \\ Baldinger and Alfred \\ Frontera are Neurologists \\ and Igor Sirotkin is a \\ Neuroradiologist, all at Bay \\ Pines VA Healthcare System \\ in Florida. \\ Correspondence: \\ Pressley Chakales \\ (pressley.chakales@gmail.com)
}

$\mathrm{H}$ emiballismus is an acquired hyperkinetic movement disorder characterized by unilateral, involuntary, often large-amplitude limb movements. Ballistic movements are now considered to be on the choreiform spectrum. ${ }^{1}$ Movements usually involve both the arm and leg, and in half of cases, facial movements such as tongue clucking and grimacing are seen. ${ }^{2,3}$ Presentations of hemiballismus vary in severity from intermittent to nearly continuous movements, which, in some cases, may lead to exhaustion, injury, or disability. Some patients are unable to ambulate or feed themselves with the affected limb.

\section{BACKGROUND}

The 2 most common causes of hemichorea-hemiballismus are stroke and hyperglycemia, with an incidence of $4 \%$ and unknown incidence, respectively. ${ }^{1,3,4}$ Other causes include HIV, traumatic brain injury, encephalitis, vasculitis, mass effect, multiple sclerosis, and adverse drug reactions ${ }^{4-7}$ Acute or subacute hemiballismus is classically attributed to a lesion in subthalamic nucleus (STN), but this is true only in a minority of cases. Hemiballismus can be caused by any abnormality in various subnuclei of the basal ganglia, including the classic location in the STN, striatum, and globus pallidus. ${ }^{4}$ Evidence shows the lesions typically involve a functional network connected to the posterolateral putamen. ${ }^{8}$

Although not commonly recognized, hyperglycemia in patients with type 2 diabetes mellitus (T2DM) is the second most common cause of hemichoreahemiballismus. ${ }^{3}$ Over the past 90 years, numerous case reports have described patients with DM with acute and subacute onset of hemiballistic and hemichoreiform movements while in a hyperglycemic state or after its resolution. Reported cases have been limited to small numbers of patients with only a few larger-scale reviews of more than 20 patients. ${ }^{79}$ Most reported cases involve geriatric patients and more commonly, females of Eastern Asian descent with an average age of onset of 71 years. ${ }^{4,10}$ Patients typically present with glucose levels from 500 to $1,000 \mathrm{mg} / \mathrm{dL}$ and hemoglobin $\mathrm{A}_{1 \mathrm{c}}\left(\mathrm{HbA}_{1 \mathrm{c}}\right)$ levels almost double the normal values. Interestingly, neuroimaging findings in these patients have consistently shown hyperintense signal in the contralateral basal ganglia on T1-weighted magnetic resonance images (MRIs). Noncontrast computed tomography (CT) shows well-defined unilateral increased density in the contralateral basal ganglia without mass effect. ${ }^{1,9,11}$

This report aims to illustrate and enhance the understanding of hemiballismus associated with hyperglycemia. One patient presented to the US Department of Veterans Affairs (VA) Bay Pines VA Healthcare System (BPVAHCS) in Florida, which motivated us to search for other similar cases. We reviewed the charts of 2 other patients who presented to BPVAHCS over the past 10 years. The first case presented with severe hyperglycemia and abnormal movements that were not clearly diagnosed as hemiballismus. MRI findings were characteristic and assisted in making the diagnosis. The second case was misdiagnosed as hemiballismus secondary to ischemic stroke. The third case was initially 

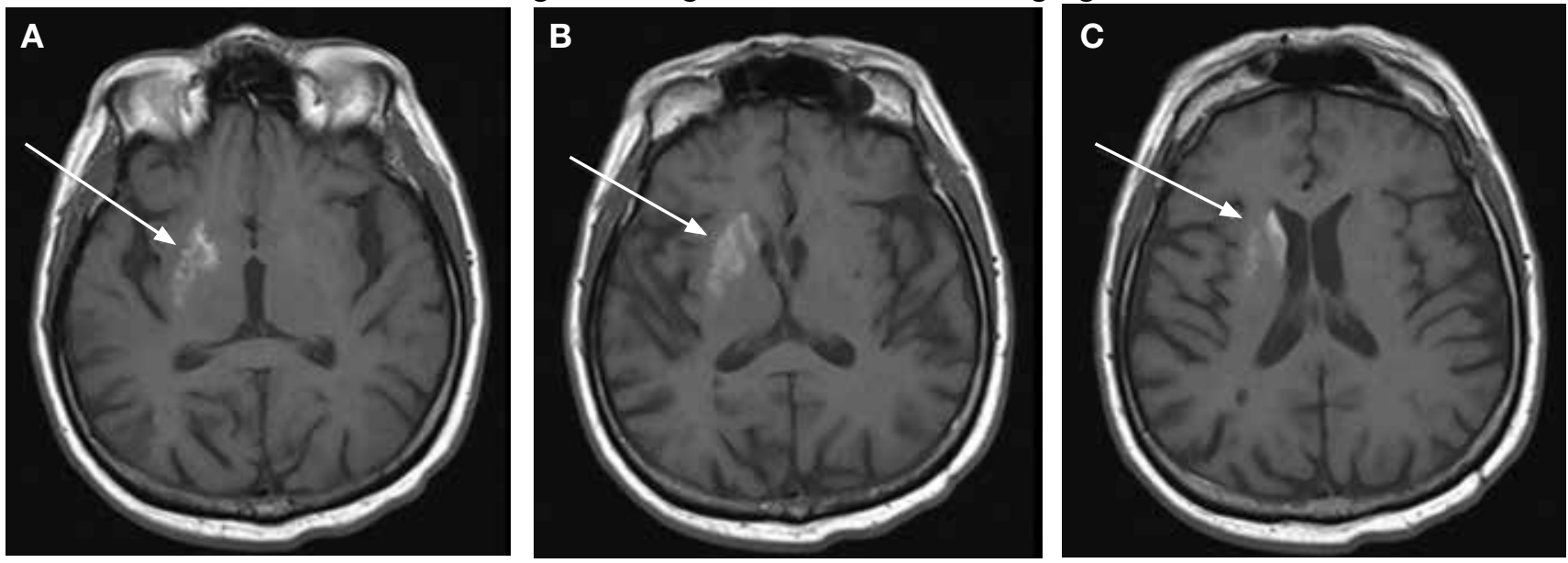

Contiguous axial slices demonstrate the varying levels of basal ganglia involvement. Arrows indicate increased signal in the right basal ganglia putamen (A), globus pallidus (B), and caudate (C).

diagnosed as conversion disorder until movements worsened and the correct diagnosis of hyperglycemia-induced hemichorea hemiballismus was confirmed by the pathognomonic neuroimaging findings.

\section{CASE PRESENTATIONS}

\section{Case 1}

A 65-year-old male with a history of uncontrolled T2DM presented with repetitive twitching and kicking movements that involved his left upper and lower extremities for 3 weeks. The patient reported that he did not take his medications or follow the recommended diabetes diet. His $\mathrm{HbA}_{1 \mathrm{c}}$ on admission was $12.2 \%$ with a serum glucose of $254 \mathrm{mg} / \mathrm{dL}$. The MRI showed a hyperintense T1 signal within the right basal ganglia including the right caudate with sparing of the internal capsule (Figure 1). There was no associated mass effect or restricted diffusion. It was compatible with a diagnosis of hyperglycemia-induced hemichorea-hemiballismus. The patient was advised to resume taking glipizide $10 \mathrm{mg}$ daily, metformin 1,000 mg by mouth twice daily, and to begin 10 units of 70/30 insulin aspart 15 minutes before meals twice daily, and to follow a low carbohydrate diet, with reduce dietary intake of sugar. At his 1-month follow-up visit, the patient reported an improvement in his involuntary movements. At the 5-month follow-up, the patient's $\mathrm{HbA}_{1 \mathrm{c}}$ level was $10.4 \%$ and his hyperkinetic movements had completely resolved.

\section{Case 2}

A white male aged 71 years with a history of T2DM, hypertension, and hyperlipidemia was admitted due to increased jerky movements in the left upper extremity. On admission, his vital signs were within normal limits and his physical examination demonstrated choreoathetoid movements with ballistic components of his left upper extremity. His laboratory results showed a glucose level of $528 \mathrm{mg} / \mathrm{dL}$ with a $\mathrm{HbA}_{1 \mathrm{c}}$ of $16.3 \%$. An initial CT obtained in the emergency department (ED) demonstrated a well-defined hyperdensity in the striatal (caudate and lentiform nucleus) region (Figure 2). There was no associated edema/mass effect that would be typical for an intracranial hemorrhage.

An MRI obtained 1 week later showed hyperintense TI signal corresponding to the basal ganglia (Figure 3). In addition, there was a questionable lacunar infarct in the right internal capsule. Due to lack of awareness regarding hyperglycemic associated basal ganglia changes, the patient's movement disorder was presumed to be ischemic in etiology. The patient was prescribed oral amantadine $100 \mathrm{mg} 3$ times daily for the hemiballismus in conjunction with treatment of his T2DM. The only follow-up occurred 5 weeks later, which showed no improvement of uncontrollable movements. Imaging at that time (not available) indicated the persistence of the abnormal signal in the right basal ganglia. This patient died later that year without further follow-up. 

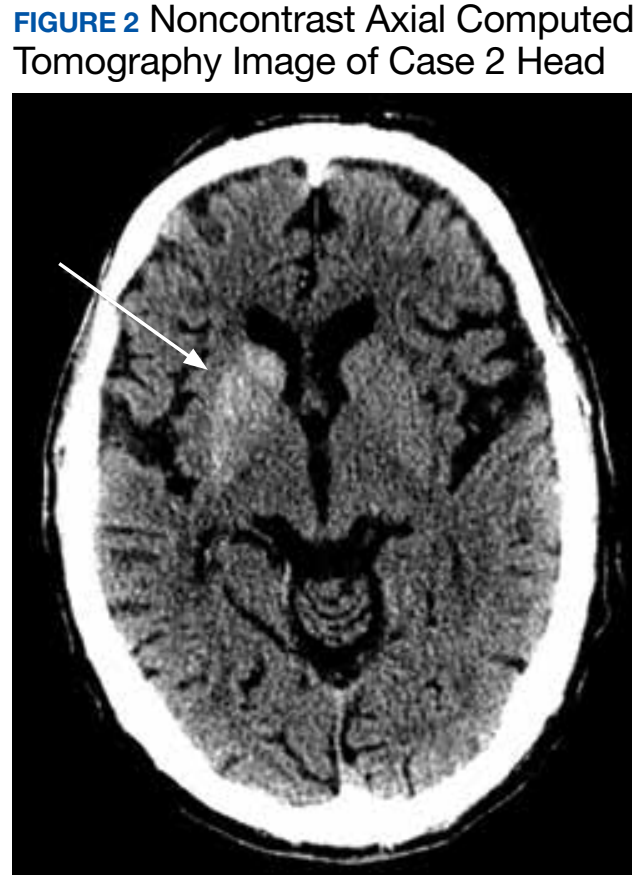

Arrow shows a well-defined region of increased attenuation in the right basal ganglia (putamen, globus pallidus, and caudate head).

\section{Case 3}

A 78-year-old white male with a history of syncope, transient ischemic attacks (TIAs), and poorly controlled T2DM presented with a 1-month history of progressively worsening involuntary, left-sided movements that began in his left shoulder and advanced to involve his arm, hand, and leg, and the left side of his face with grimacing and clucking of his tongue. Three weeks earlier, the patient had been discharged from the ED with a diagnosis of conversion disorder particularly because he experienced decreased movements when given a dose of Vitamin D. It was overlooked that administration of haloperidol had occurred a few hours before, and because the sounds made by his tongue were not felt to be consistent with a known movement disorder. A MRI of the brain was read as normal.

The patient returned 3 weeks later (the original presentation) due to his inability to perform activities of daily living because of his worsening involuntary movements. On admission, his $\mathrm{HbA}_{1 \mathrm{c}}$ was $11.1 \%$ and his glucose was $167 \mathrm{mg} / \mathrm{dL}$. On chart review, it was revealed that the patient's $\mathrm{HbA}_{1 \mathrm{c}}$ had been $>9 \%$ for the past 3 years with an increase from $10.1 \%$ to $11.1 \%$ in the 3 months pre-
FIGURE 3 Noncontrast Axial T1-Weighted Magnetic Resonance Image of Case 2 Head

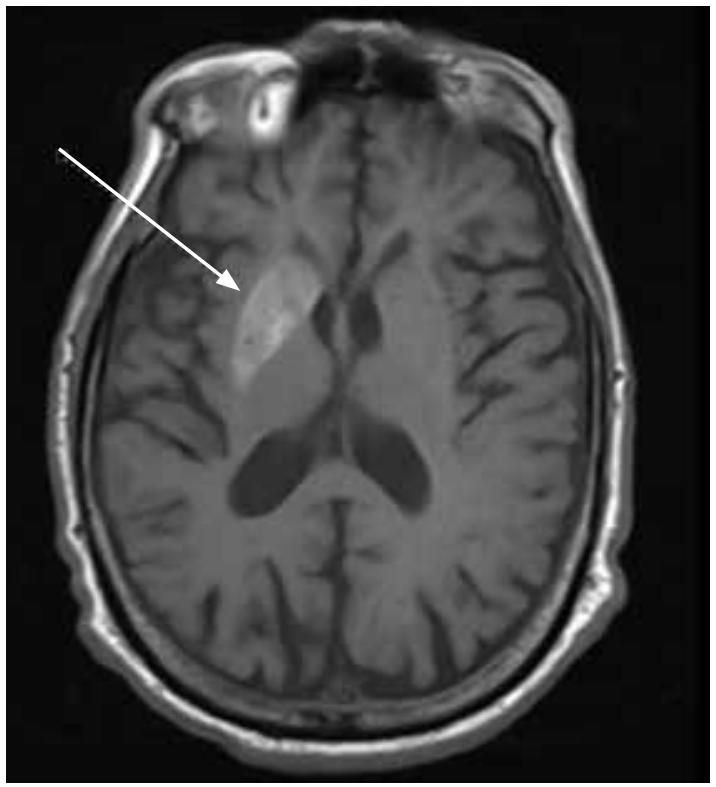

Arrow shows a well-defined region of increased signal in the right basal ganglia.

ceding the onset of his symptoms.

On admission a MRI showed a unilateral right-sided $\mathrm{T} 1$ hyperintensity in the basal ganglia, no acute ischemia (Figure 4). In retrospect, subtle increased T1 signal can be seen on the earlier MRI (Figure 5). In view of the patient's left-sided symptoms, DM, and MRI findings, a diagnosis of hyperglycemia-induced hemichorea-hemiballismus was made as the etiology of the patient's symptoms.

The patient was prescribed numerous medications to control his hyperkinesia including (and in combination): benztropine, gabapentin, baclofen, diphenhydramine, benzodiazepines, risperidone, olanzapine, and valproic acid, which did not control his movements. Ultimately, his hyperglycemic hemiballismus improved with tight glycemic control and oral tetrabenazine $12.5 \mathrm{mg}$ twice daily. This patient underwent a protracted course of treatment with 17 days of inpatient medical admission, 3 weeks inpatient rehabilitation, and subsequent transfer to an assisted living facility.

\section{DISCUSSION}

The 3 cases presented in this report contribute to the evidence that severe 

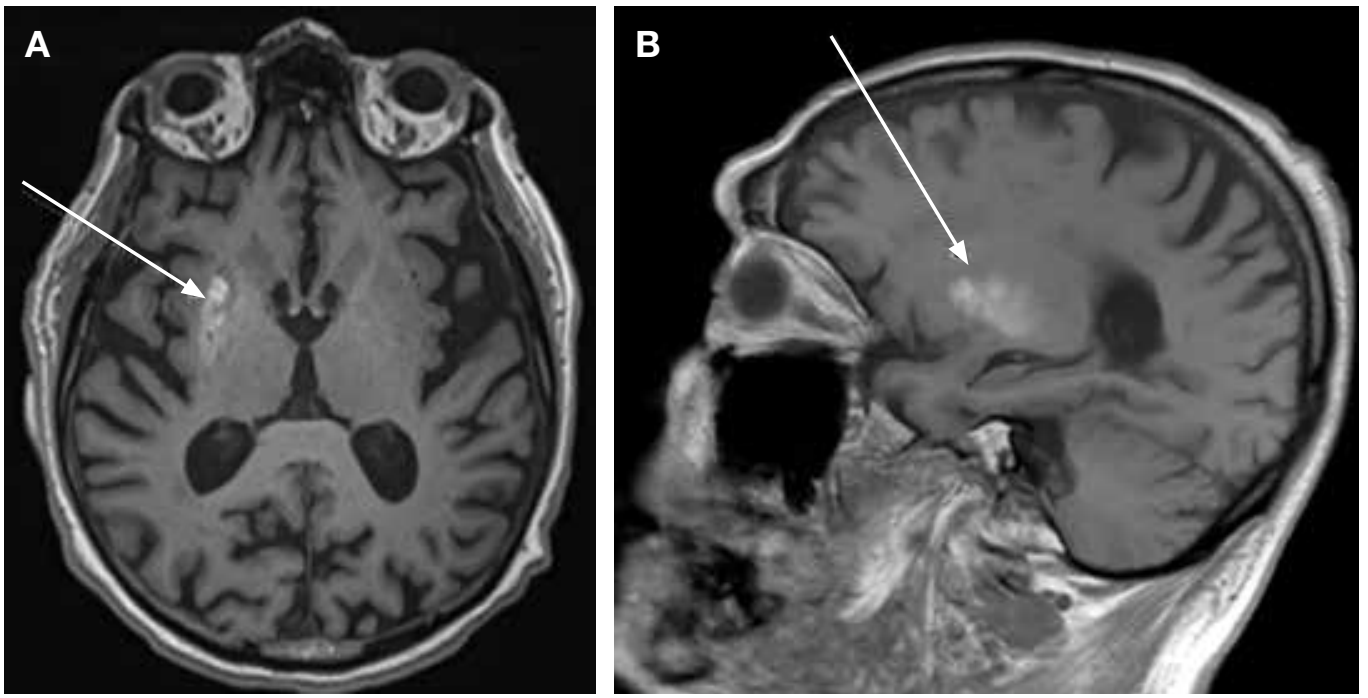

A, axial image, arrow shows a well-defined region of increased signal in the right basal ganglia. B, sagittal image, arrow shows increased signal in the right basal ganglia.

\section{FIGURE 5 Noncontrast Axial and Sagittal T1-Weighted Magnetic Resonance Imaging of Case 3 Head}
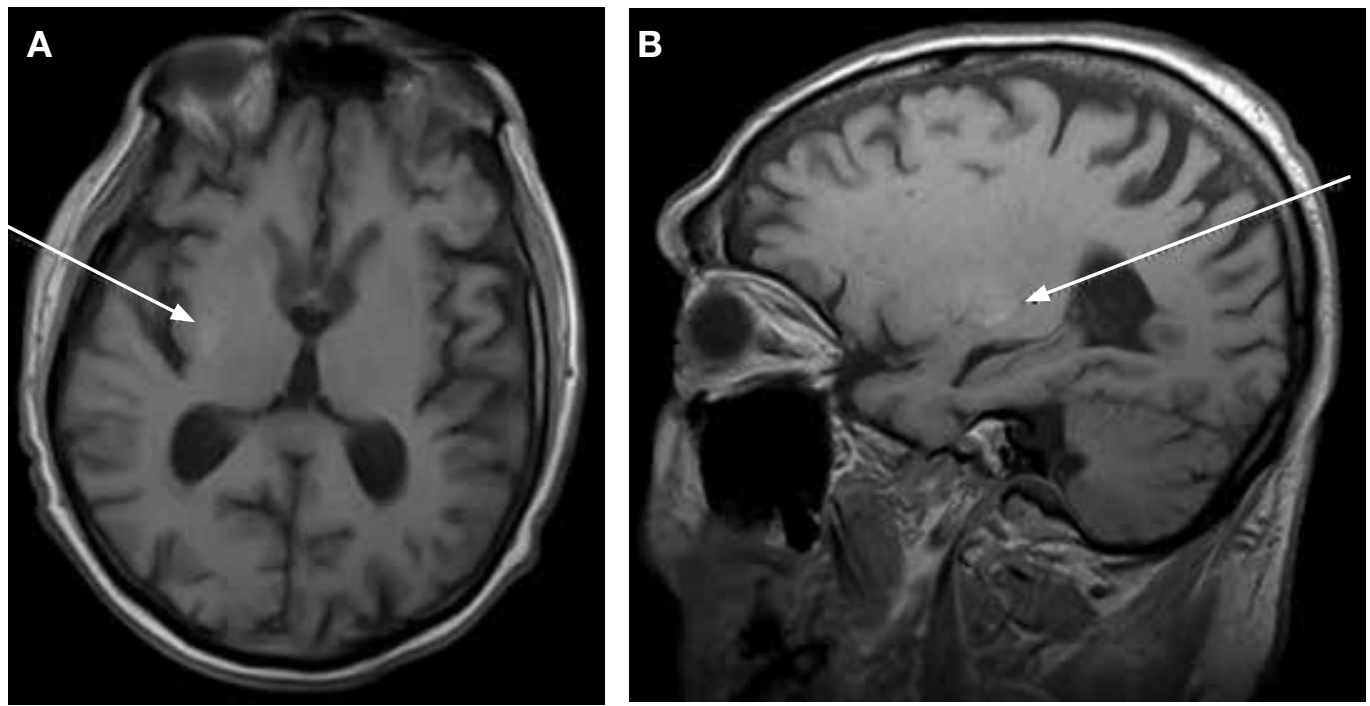

Arrows show the subtle increased signal in the right basal ganglia can be seen about 1 month earlier in both axial (A) and sagittal (B) images.

persistent hyperglycemia can result in movement disorders that mimic those seen after basal ganglia strokes. As with Case 2, past literature describes many cases of acute hyperglycemic episodes with glucose ranging from 500 to $1,000 \mathrm{mg} / \mathrm{mL}$ presenting with hemiballismus. ${ }^{1,3}$ However, there are many cases that describe hemiballismus occurring after glycemic correction, persisting despite glycemic correction, and presenting without an acute hyperglycemic episode, but in the setting of elevated $\mathrm{HbA}_{1 \mathrm{c}}$, as in Case 3. ${ }^{12,13}$ Notably, all 3 cases in this series had marked elevation in their $\mathrm{HbA}_{1 \mathrm{c}}$ levels, which suggests that a more chronic hyperglycemic state or multiple shorter periods of hyperglycemia may be necessary to produce the described hyperkinetic movements.

Case reports describe the pathognomonic T1 hyperintensity of the basal ganglia that is identified in all 3 cases presented here. While the exact etiology remains unclear, the 
hyperintensity is hypothesized to be related to metabolic derangements caused by hyperviscosity of the blood in the small end arteries feeding the basal ganglia.,11 These abnormalities in turn interrupt the signaling cascade with abnormal firing rates or firing patterns, leading to reduced inhibition of the motor thalamus and ultimately present as hemiballismus. ${ }^{1,3,7}$ While most cases presented with unilateral hyperkinesis and associated contralateral basal ganglia abnormalities, there are reports of both unilateral and bilateral movements associated with bilateral basal ganglia hyperintensities on imaging. ${ }^{9}$ The predilection for unilateral brain lesions may be explained by the varying degree of small vessel disease in different areas of the brain leading to perfusion deficits worsened by hyper viscosity. Further research into this is required to elucidate the exact pathophysiologic mechanism.

The course of disease for patients ranges from resolution within hours of tight glycemic control to persistent movements for $>3$ months with a gradual improvement in severity. ${ }^{12,13}$ Treatments center on the importance of tight glycemic control to protect against the protracted course described in Case 3. Swift recognition of this rare condition is critical because improved glycemic control decreases the severity and duration of this disease. The significant disability associated with Case 3 highlights the need for prompt recognition and early, aggressive glycemic management to prevent the progression of hemiballismus. In addition to glycemic control, various CNS medications such as typical and atypical antipsychotics and tetrabenazine are firstline therapy with chemodenervation and surgical lesioning in cases unresponsive to medication therapy.

When unrecognized, hyperglycemic hemiballismus is associated with significant morbidity and mortality. The patients presented in this report were subject to either delayed diagnosis or misdiagnosis as stroke or psychiatric disorder. The rarity of the disorder, lack of evidence delineating pathogenesis and causality, low level of awareness, and varying presentations of patients all contribute to the challenge of recognizing, diagnosing, and treating hemiballismus due to hyperglycemia. This challenge can subsequently result in deteriorating symptoms, prolonged hospital stays, and unnecessary health care costs.

\section{CONCLUSIONS}

While hemiballismus due to severe persistent hyperglycemia is rare, the goal of this report is to highlight its occurrence in patients with T2DM. Further research can help develop a standardized, effective treatment strategy for these patients. Currently, lowering and maintaining appropriate glucose and $\mathrm{HbA}_{1 \mathrm{c}}$ levels is the most effective treatment approach. Potential areas of research include alternative medical and surgical treatment interventions for patients while glycemic control is being achieved or for those who fail to benefit from glycemic control alone. Some success has been demonstrated with the use of antidopaminergic medications such as atypical antipsychotics and tetrabenazine and these medications should be considered when tight, sustained glycemic control alone is not successful in treating this disorder in the acute stages. Hopefully, with increasing awareness and recognition of hemiballismus related to hyperglycemia, more large-scale clinical trials can be conducted that will result in an effective treatment strategy for this devastating disorder.

\section{Acknowledgments}

We thank Mahlon DeLong, MD, for his comments on the manuscript.

\section{Author disclosures}

The authors report no actual or potential conflicts of interest with regard to this article.

\section{Disclaimer}

The opinions expressed herein are those of the authors and do not necessarily reflect those of Federal Practitioner, Frontline Medical Communications Inc., the US Government, or any of its agencies. This article may discuss unlabeled or investigational use of certain drugs. Please review the complete prescribing information for specific drugs or drug combinations-including indications, contraindications, warnings, and adverse effects-before administering pharmacologic therapy to patients.

\section{References}

1. Hawley JS, Weiner WJ. Hemiballismus: current concepts and review. Parkinsonism Relat Disord. 2012;18(2):125-129. doi:10.1016/j.parkreldis.2011.08.015

2. Gasca-Salas C, Lang AE. Paroxysmal Hemiballism/ Hemichorea Resulting from Transient Ischemic Attacks. Mov Disord Clin Pract. 2015;3(3):303-305. doi:10.1002/mdc3.12268

3. Garcia-Grimshaw MA, Jimenez-Ruiz A, Ornelas-Velazquez A, Luna-Armenta A, Gutierrez-Manjarrez FA. New-onset diabetes presenting as monoballism secondary to a mixed hyperglycemic crisis. Cureus. 2018;10(6):e2882 . doi:10.7759/cureus.2882 
4. Postuma RB, Lang AE. Hemiballism: revisiting a classic disorder. Lancet Neurol. 2003;2(11):661-668. doi:10.1016/s1474-4422(03)00554-4

5. Gallo BV, Shulman LM, Weiner WJ, Petito CK, Berger JR. HIV encephalitis presenting with severe generalized chorea. Neurology. 1996;46(4):1163-1165. doi:10.1212/wnl.46.4.1163

6. Provenzale JM, Glass JP. Hemiballismus: CT and MR findings. J Comput Assist Tomogr. 1995;19(4):537-540.

7. Hodde M, Rowe KE, Surapaneni K, Terrigno P, Brighenti A, Altschuler EL. Management of severe hemiballismus: treatment challenges in the acute inpatient rehabilitation setting: a case presentation. PMR. 2017;9(7):732-735 doi:10.1016/j.pmrj.2016.10.023

8. Laganiere S, Boes AD, Fox MD. Network localization of hemichorea-hemiballismus. Neurology. 2016;86(23):2187-2195.

doi:10.1212/WNL.0000000000002741

9. Cosentino C, Torres L, Nuñez Y, Suarez R, Velez $M$, Flores M. Hemichorea/hemiballism associated with hyperglycemia: report of 20 cases.
Tremor Other Hyperkinet Mov (NY). 2016;6:402. doi:10.7916/D8DN454P

10. Oh SH, Lee KY, Im JH, Lee MS. Chorea associated with non-ketotic hyperglycemia and hyperintensity basal ganglia lesion on T1-weighted brain MRI study: a metaanalysis of 53 cases including four present cases. $J$ Neurol Sci. 2002;200(1-2):57-62.

doi:10.1016/s0022-510x(02)00133-8

11. Carrion DM, Carrion AF. Non-ketotic hyperglycaemia hemichorea-hemiballismus and acute ischaemic stroke. BMJ Case Rep. 2013;2013:bcr2012008359. doi:10.1136/bcr-2012-008359

12. Cho HS, Hong CT, Chan L. Hemichorea after hyperglycemia correction: a case report and a short review of hyperglycemia-related hemichorea at the euglycemic state. Medicine (Baltimore). 2018;97(10):e0076. doi:10.1097/MD.0000000000010076

13. Lin YC, Lin YC. Prolonged hemiballism after the remission of non-ketotic hyperosmolar syndrome. BMJ Case Rep. 2012;2012:bcr0120125627. doi:10.1136/bcr.01.2012.5627 